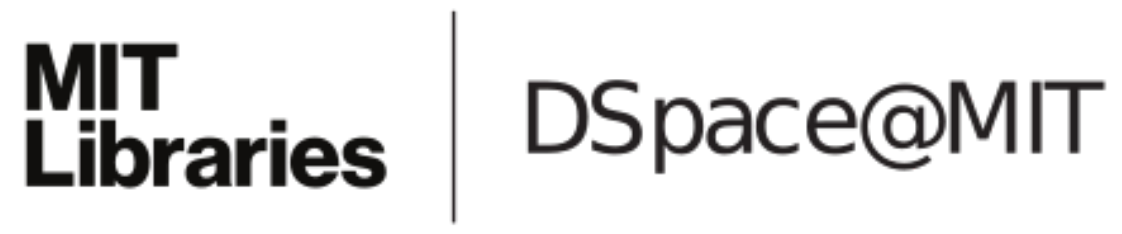

\author{
MIT Open Access Articles
}

Scaling Effects on Single-Event Transients in InGaAs FinFETs

The MIT Faculty has made this article openly available. Please share how this access benefits you. Your story matters.

Citation: Gong, Huiqi et al. "Scaling Effects on Single-Event Transients in InGaAs FinFETs." IEEE Transactions on Nuclear Science, 65, 1 (January 2018): 296 - 303 @ 2018 IEEE

As Published: http://dx.doi.org/10.1109/tns.2017.2778640

Publisher: Institute of Electrical and Electronics Engineers (IEEE)

Persistent URL: https://hdl.handle.net/1721.1/126203

Version: Author's final manuscript: final author's manuscript post peer review, without publisher's formatting or copy editing

Terms of use: Creative Commons Attribution-Noncommercial-Share Alike 


\title{
Scaling Effects on Single-Event Transients in InGaAs FinFETs
}

\author{
Huiqi Gong, Student Member, IEEE, Kai Ni, Student Member, IEEE, En Xia Zhang, Senior Member, IEEE, \\ Andrew L. Sternberg, Member, IEEE, John A. Kozub, Kaitlyn L. Ryder, Ryan F. Keller, Landen D. Ryder, \\ Sharon M. Weiss, Senior Member, IEEE, Robert A. Weller, Senior Member, IEEE, \\ Michael L. Alles, Member, IEEE, Robert A. Reed, Fellow, IEEE, Daniel M. Fleetwood, Fellow, IEEE, \\ Ronald D. Schrimpf, Fellow, IEEE, Alon Vardi, Member, IEEE, Jesús A. del Alamo, Fellow, IEEE
}

\begin{abstract}
The single-event-transient response of InGaAs FinFETs with different fin widths is examined using pulsed-laser and heavy-ion irradiation. Devices with wider fins collect more charge in both environments. Quantum-well structures confine charge collection in the channel, and determine the sensitive volume. Simulations show that the charge density produced by irradiation is similar for devices with different fin widths, but more charge is collected by wider fin devices due to the larger channel volume. Charge accumulated in the buffer and substrate layers modulates the body potential, altering the degree of back-gate control, leading to additional effects associated with charge accumulation in wider fin devices. Optical simulations for a model system suggest that optical phenomena in the fins should be considered for laser testing. These include optical interference, plasmonic enhancement at the metal-dielectric interfaces, and enhanced electron-hole pair recombination due to multiple reflections in multi-gate devices with nanoscale dimensions.
\end{abstract}

Index Terms - InGaAs, FinFETs, single-event transient, pulsed-laser, heavy-ion, fin width, scaling effects, charge collection, technology computer-aided design (TCAD)

\section{INTRODUCTION}

$\mathrm{I}^{\mathrm{n}}$ ncorporation of compound semiconductors is a promising candidate to address scaling challenges beyond the $7 \mathrm{~nm}$ node due to their outstanding electron transport properties [1]. Their high electron mobility and high frequency response make these devices an ideal option for many space applications [1]-[3]. A significant amount of research focuses on single-event effects (SEE) in these devices [2]-[8]. Most of these works consider III-V planar MESFETs and HEMTs. Only limited work describing the SEE response of III-V FinFETs has been reported. InGaAs and Ge complementary FinFET technology is promising for next generation NMOS/PMOS FETs. Recent work suggests that SEE in circuits built in this tech-

Manuscript received July 14, 2017; revised October 9, 2017; accepted XX. Date of publication XX; date of current version XX. This work was supported by the Defense Threat Reduction Agency through its fundamental research program (contract \#HDTRA 1-14-1-0057).

H. Gong, K. Ni, E. X. Zhang, A. L. Sternberg, K. L. Ryder, R. F. Keller, L. D. Ryder, S. M. Weiss, R. A. Weller, M. L. Alles, R. A. Reed, D. M. Fleetwood, and R. D. Schrimpf are with the Department of Electrical Engineering and Computer Science, Vanderbilt University, Nashville, TN 37235 USA (e-mail: huiqi.gong@vanderbilt.edu).

J. A. Kozub is with the Department of Physics, Vanderbilt University, Nashville, TN 37235, USA.

A. Vardi and J. A. del Alamo are with Microsystems Technology Laboratories, Massachusetts Institute of Technology, Cambridge, MA 02139, USA. nology are likely to be dominated by ion strikes on the InGaAs FinFET [9].

Fin geometry is optimized for device electrical performance and manufacturability of a given process. Dimensions can scale as process generations scale, and vary among manufacturers and even within a single process [1]. From the 22-nm technology node to the 10-nm technology node, fin height increases while fin width decreases [10]. The gate length dependence of single-event transient (SET) response of InGaAs FinFETs has been evaluation by $\mathrm{Ni}$ et al. using a high-speed pulsed-laser system [11]. The peak drain current was found to increase as the gate length decreased, which suggests that parasitic bipolar action is fully suppressed in longer channel devices [11]. In this work, we have evaluated the impact of fin width on the SET response of InGaAs FinFETs via pulsed-laser and heavy-ion irradiation. Over all, the trends observed in pulsed-laser irradiation are consistent with those of heavy-ion irradiation. However, there are a few differences. In both situations, devices with wider fins collect more charge, due to their greater sensitive volumes. Charge accumulated in the buffer and substrate modulates the body potential, leading to additional charge collection in wider fin devices. Finite-difference time-domain (FDTD) simulations for a model system carried out using Lumerical [12] show that optical intensity is modulated inside narrow fins during laser irradiation. As technology scales, this optical modulation is important to consider.

\section{DEVICE STRUCTURE AND EXPERIMENTAL SETUP}

The device under test is a double-gate InGaAs FinFET [13]. A 3D qualitative view of the device is shown in Fig. 1c; layers are not drawn to scale. Details of the fin structure are shown in Fig. 1a. Fig. 1b shows a cross-sectional diagram along the fin width direction of a single fin structure. There are 11 fins in parallel for each device, with $0.8 \mu \mathrm{m}$ pitch between fins. The height of each fin is $220 \mathrm{~nm}$, with a $40 \mathrm{~nm}$ thick $\operatorname{In}_{0 .}{ }_{53} \mathrm{Ga}_{0.47} \mathrm{As}$ channel layer, and the gate length is $100 \mathrm{~nm}$ for all devices reported here. Under the channel, a $0.4-\mu \mathrm{m} \mathrm{In}_{0.52} \mathrm{Al}_{0.48} \mathrm{As}$ buffer layer is grown on a $600-\mu \mathrm{m}$ semi-insulating InP substrate. 40 $\mathrm{nm}$ of $\mathrm{SiO}_{2}$ serves as a hard mask, which electrostatically decouples the top gate from the channel. As a result, devices are only controlled by the two side gates. A 5-nm $\mathrm{Al}_{2} \mathrm{O}_{3}$ gate dielectric is deposited by atomic layer deposition. A silicon delta doping layer $5 \mathrm{~nm}$ below the channel is used to enhance channel electron density. The detailed fabrication process is described 
in [14]. Figs. $2 \mathrm{a}$ and $2 \mathrm{~b}$ show the energy band diagrams in the fin width and fin height directions, respectively.

Devices with different fin widths were tested with both pulsed-laser and heavy-ion irradiations. A tunable wavelength laser system and high resolution transient capture system are used for laser testing [11]. The experimental setup for heavy-ion irradiation is described in [8]. The pulsed-laser testing experiments were performed at Vanderbilt University. The heavy-ion experiments were performed with the 88 inch cyclotron at Lawrence Berkeley National Laboratory (LBNL). This paper significantly extends the initial results on InGaAs FinFETs by Ni, et al. [11].

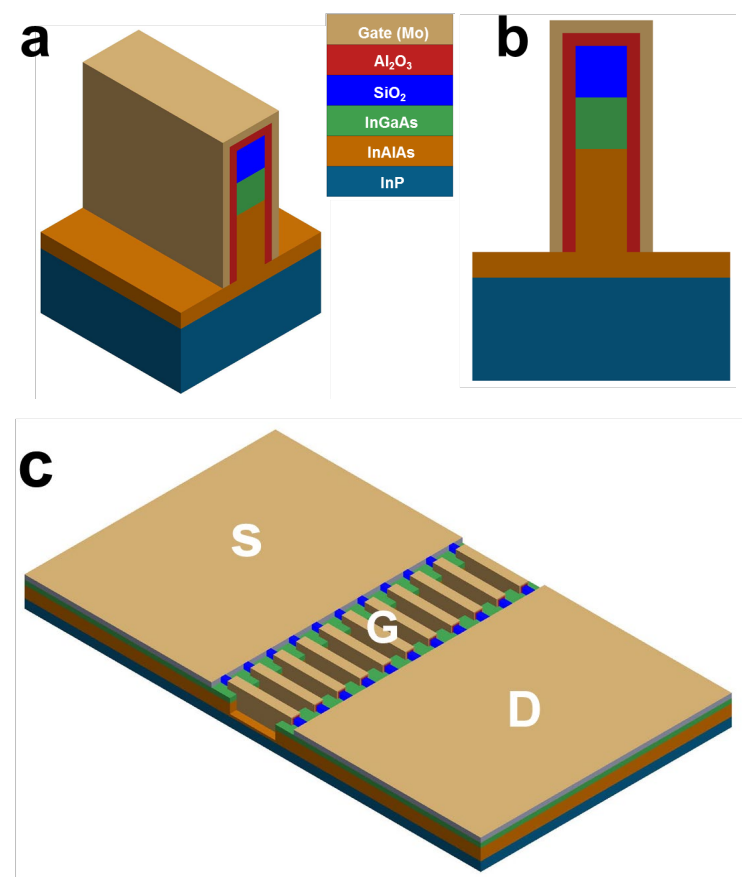

Fig. 1. (a) $3 \mathrm{D}$ qualitative representation of the fin structure of the InGaAs double-gate FinFETs, (b) cross-section along the fin width direction, and (c) 3D schematic diagram of 11 fin InGaAs double-gate FinFETs. Gate (G), drain (D), and source (S) are labeled on the diagram. (Not drawn to scale) a

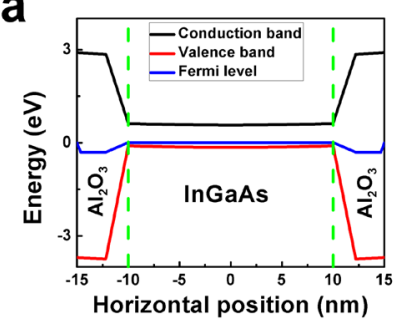

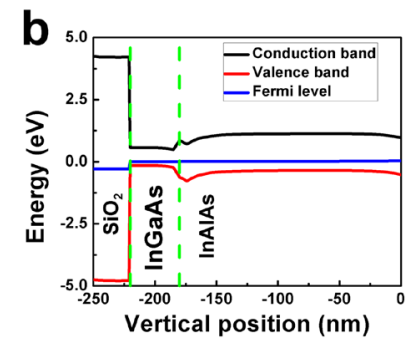

Fig. 2. Band diagrams cut through the fin structure along the (a) fin width direction and (d) fin height direction.

\section{EXPERIMENTAL RESUlTS AND DISCUSSION}

\section{A. Pulsed-laser induced charge generation}

The pulsed-laser wavelength used in the experiment is 1260 $\mathrm{nm}$, corresponding to a photon energy of $0.98 \mathrm{eV}$, which is above the band gap of $\operatorname{In}_{0.53} \mathrm{Ga}_{0.47}$ As. Table 1 lists the charge generation mechanisms in the materials used in the devices.
Both two-photon absorption (TPA) and single-photon absorption (SPA) mechanisms contribute to charge generation in the InGaAs channel, while only TPA contributes to charge generation in the InAlAs and InP layers [11].

TABLE I CHARGE GENERATION MECHANISM FOR 1260 NM LASER

\begin{tabular}{ccc}
\multicolumn{2}{c}{ TABLE I CHARGE GENERATION MECHANISM FOR 1260 NM LASER } \\
\hline \hline Material & Band gap $(\mathrm{eV})$ & $\begin{array}{c}\text { Photon absorption } \\
\text { mechanism }\end{array}$ \\
\hline $\mathrm{In}_{0.53} \mathrm{Ga}_{0.47} \mathrm{As}$ & 0.75 & SPA/TPA \\
$\mathrm{In}_{0.52} \mathrm{Al}_{0.48} \mathrm{As}$ & 1.46 & TPA \\
$\mathrm{InP}$ & 1.35 & TPA \\
\hline \hline
\end{tabular}

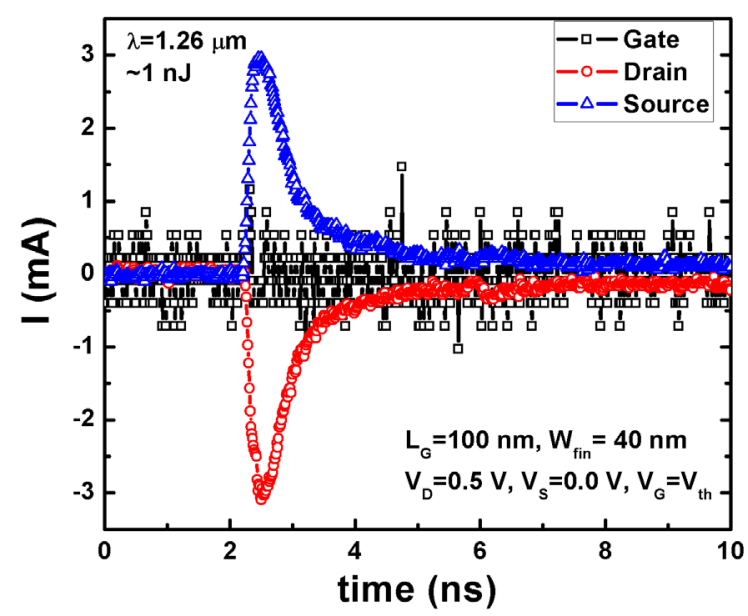

Fig. 3. SET captured during TPA testing for an InGaAs FinFET. $L_{G}=100 \mathrm{~nm}$, $W_{f}=40 \mathrm{~nm}$. Initial results were obtained with a pulsed-laser system with tunable wavelength [11]; this work extends the experiments and analysis.

Fig. 3 shows a typical laser-induced transient for a pulse delivered to the center of a device with fin width of $40 \mathrm{~nm}$ under a bias condition of $V_{D}=0.5 \mathrm{~V}, V_{S}=0 \mathrm{~V}$, and $V_{G}=V_{t h}$, where $V_{t h}$ is the threshold voltage of the devices with initial value in the range of $-0.1 \pm 0.1 \mathrm{~V}$. The spot size of the incident Gaussian beam is approximately $1.2 \mu \mathrm{m}$, as determined via a typical knife-edge measurement at the beam waist [15]. The maximum peak current is achieved when the center (maximum intensity) of the laser spot is located on the fin. The radius of the spot $(0.6 \mu \mathrm{m})$ is smaller than the fin pitch $(0.8 \mu \mathrm{m})$, which localizes the irradiation on a single fin. Transient signals are recorded mainly on the drain and source; the peak gate current is negligible. This occurs because, across the fin width direction, the InGaAs channel and $\mathrm{Al}_{2} \mathrm{O}_{3}$ gate dielectric form a deep type-I quantum-well. Along the fin height direction, the InGaAs channel, InAlAs buffer, and $\mathrm{SiO}_{2}$ also form a type-I quantum-well, as shown in Figs. 2a and b. Thus, carriers generated by irradiation are confined in the channel layer once they have been collected. This makes the channel layer critical to the charge collection. The transients on drain and source are symmetric for various strike locations, which suggests the transient current comes mainly from channel collection. There is a tail (compared with the prompt peak current) on the transient signal, which is consistent with previous work on planar III-V MOSFETs [8] and FinFETs [11]. The tail current in Fig. 2 is mainly caused by charge collection from the substrate. Charge generated more deeply within the substrate leads to a longer time for carriers to transport into the channel [11]. This 
response differs significantly from bulk Si FinFETs, where no tail current is observed within the transient signal [16]. This is because most deposited charge in the substrate of a Si bulk FinFET is collected by the substrate contact through the conductive substrate, instead of the source or drain. All peak drain currents in this paper are reported as absolute values. For both laser and heavy ion tests, the total collected charge is based on integration of current vs. time beginning and ending at $10 \%$ of the peak value.
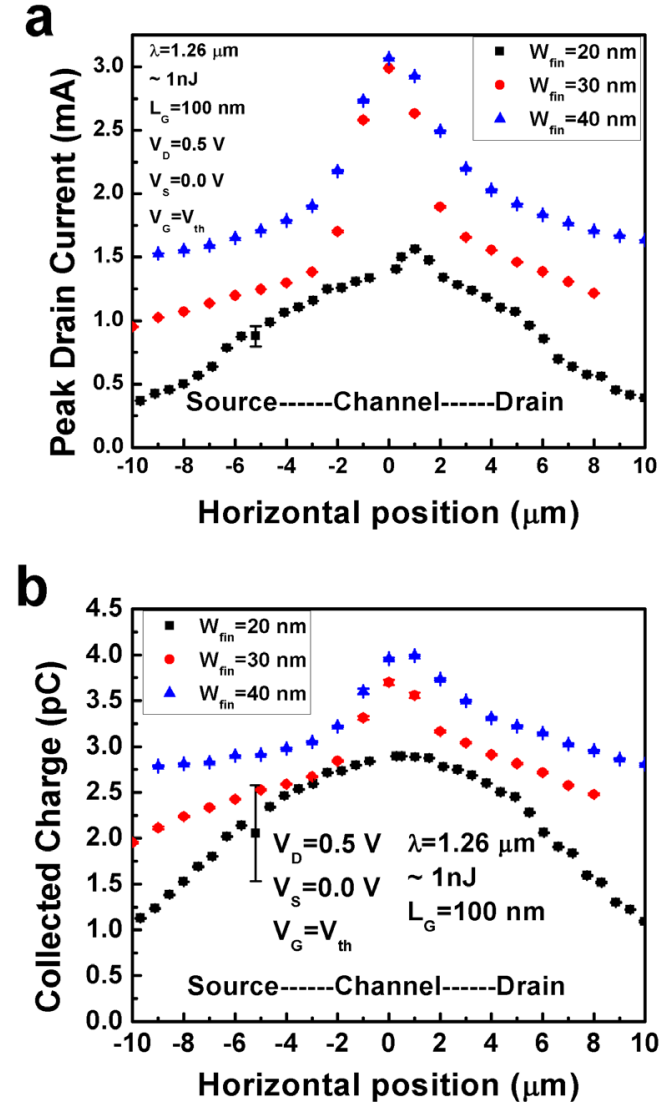

Fig. 4. Peak drain current (a) and drain collected charge (b) along a line scan for InGaAs FinFETs of different fin widths during TPA testing.

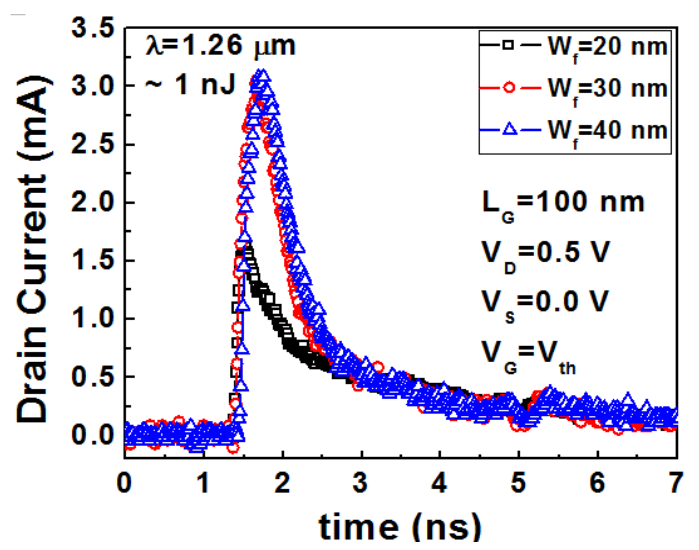

Fig. 5. Drain current transients for InGaAs FinFETs of different fin widths during TPA testing (peak drain current is taken as absolute value). The laser strike is at the center of the gate.

Figs. 4a and b show peak drain current and collected charge along a line scan from source to drain for devices with different fin widths. There is some spatial asymmetry; the peak drain current is higher when the laser is on the drain side, due to the higher electric field on the drain side. Both the peak drain currents and collected charge for narrower fin devices $\left(W_{f}=20\right.$ $\mathrm{nm})$ are much smaller compared with wider fin devices $\left(W_{f}=\right.$ $30 \mathrm{~nm}$ and $40 \mathrm{~nm}$ ).

A comparison of drain current transients for devices with different fin widths is shown in Fig. 5. For the $20 \mathrm{~nm}$ fin width device, peak currents are much smaller than for $30 \mathrm{~nm}$ or $40 \mathrm{~nm}$ fin widths. The tails of the transients overlap for all three devices, because all devices have the same substrate material and geometry, leading to similar charge collection from the substrate in all cases. Charge collection of the charge injected in the channel produces the prompt increase in current [11]. Even though all transients have similar tails, the integrated collected charge is much smaller for the $20 \mathrm{~nm}$ devices due to the lower peak current.

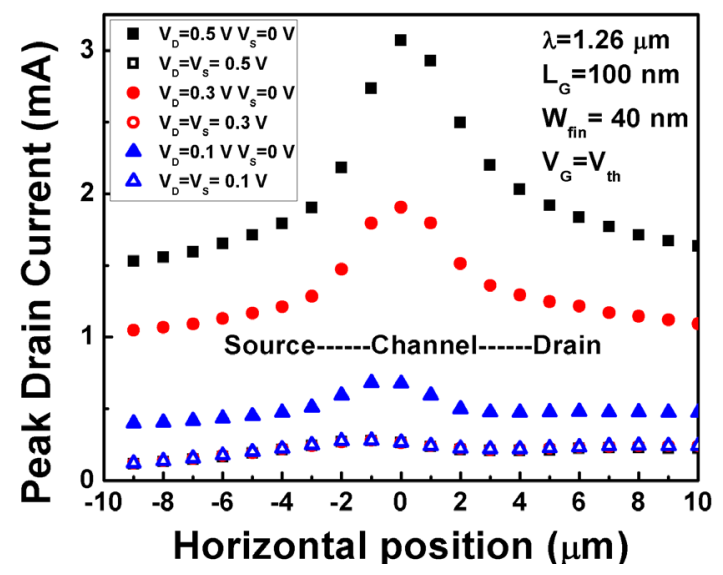

Fig. 6. Peak drain current along a line scan at different drain bias during TPA testing. $W_{f}=40 \mathrm{~nm}, L_{G}=100 \mathrm{~nm}$.

The peak drain current along a line scan at different drain biases is shown in Fig. 6. Higher drain bias produces a stronger electric field, resulting in higher peak drain current. When the drain and source are biased at the same potential, the electric field along the channel is a minimum. The peak drain current is much lower when the source and drain are tied together. This is consistent with previous studies in InGaAs FinFETs [11], and differs significantly from $\mathrm{Si}$ and SiGe FinFETs, for which larger charge collection occurs typically at the S/D junctions [16]-[18]. Hence, the detailed nature of the gate materials and/or presence or absence of quantum well structures can fundamentally alter the charge collection in FinFETs based on compound semiconductor materials, causing parasitic bipolar effects [11].

\section{B. Heavy-ion induced charge generation}

The heavy ions used in this experiment are Xeon, Krypton, Argon, and Neon. Table 2 lists the linear energy transfer (LET) in $\mathrm{In}_{0.53} \mathrm{Ga}_{0.47} \mathrm{As}$ and energy of these ions.

Fig. 7 shows typical heavy-ion induced transients for a device with fin width of $40 \mathrm{~nm}$ biased at $V_{D}=0.5 \mathrm{~V}, V_{S}=0 \mathrm{~V}$, and $V_{G}=V_{t h}$. The peak current produced by the $40 \mathrm{MeV} \cdot \mathrm{cm}^{2} / \mathrm{mg} \mathrm{Xe}$ ion is similar to that measured in previous studies [8] of 
heavy-ion induced charge collection in planar InGaAs MOSFETs irradiated with $14.3 \mathrm{MeV}$ oxygen ions, which has a lower LET of $3.9 \mathrm{MeV} \cdot \mathrm{cm}^{2} / \mathrm{mg}$ in $\operatorname{In}_{0.53} \mathrm{Ga}_{0.47}$ As. This suggests that planar InGaAs MOSFETs are more sensitive to SET than FinFETs due to the larger sensitive volumes for planar devices.

TABLE II. IONS THAT COMPRISE THE LBNL $10 \mathrm{MEV/U} \mathrm{COCKTAIL}$

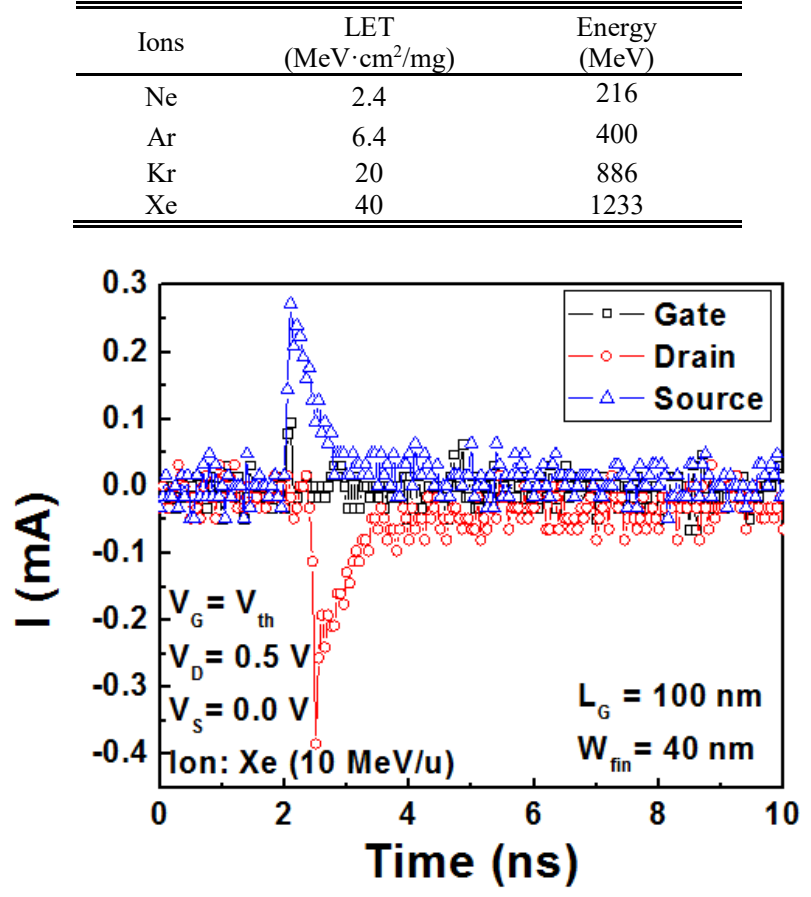

Fig. 7 SET captured during heavy ion testing for an InGaAs FinFET. $L_{G}=100$ $\mathrm{nm}, W_{f}=40 \mathrm{~nm}$.

The heavy-ion induced transient currents in Fig. 7 are much smaller than the laser-induced transients in Figs. 3 and 5 because the laser pulse energy used generates a larger amount of charge in and near the sensitive volume than that generated by these particular ions. There is asymmetry on the drain and source transient signals, where the peak drain current shows a delay, compared with the peak source current. In contrast, there is no delay in the pulsed-laser induced SET. This is mainly due to the limited oscilloscope temporal resolution (50 ps) for the heavy ion tests rather than any device-level effects. In Fig. 7, a single data point is recorded on the rising edge, which may miss the real peak value. All laser tests are done with an oscilloscope with time resolution of $12.5 \mathrm{ps}$, which is capable of more precise peak current measurement than that of the oscilloscope available for the remote-site heavy ion testing. The time delay is also likely affected by the signal paths being slightly different, caused by variation in cable length and feedthroughs.

Fig. 8a shows collected charge vs. peak drain current for 10 $\mathrm{MeV} / \mathrm{u}$ Xenon ion irradiation of devices with different fin widths. Each data point is averaged from 50 transient events. The $20 \mathrm{~nm}$ fin-width device shows much less charge collection than the $30 \mathrm{~nm}$ and $40 \mathrm{~nm}$ fin width devices, consistent with the pulsed-laser results. The peak current is independent of fin width, which is different from the pulsed-laser results. This result is most likely caused by differences between spatial and temporal charge distributions generated by pulsed-laser and heavy-ion irradiation. For example, in pulsed laser testing, a large amount of charge is generated at the focal point. In heavy-ion testing, much less charge is generated in surrounding regions of the device. Moreover, the optical phenomena discussed in Section V for laser irradiation do not apply in ion testing. Fig. 8b shows collected charge and peak drain current for a $40 \mathrm{~nm}$ device at different drain biases. Higher drain bias leads to higher peak drain current. There is no transient observed if the drain and source are biased at the same potential.
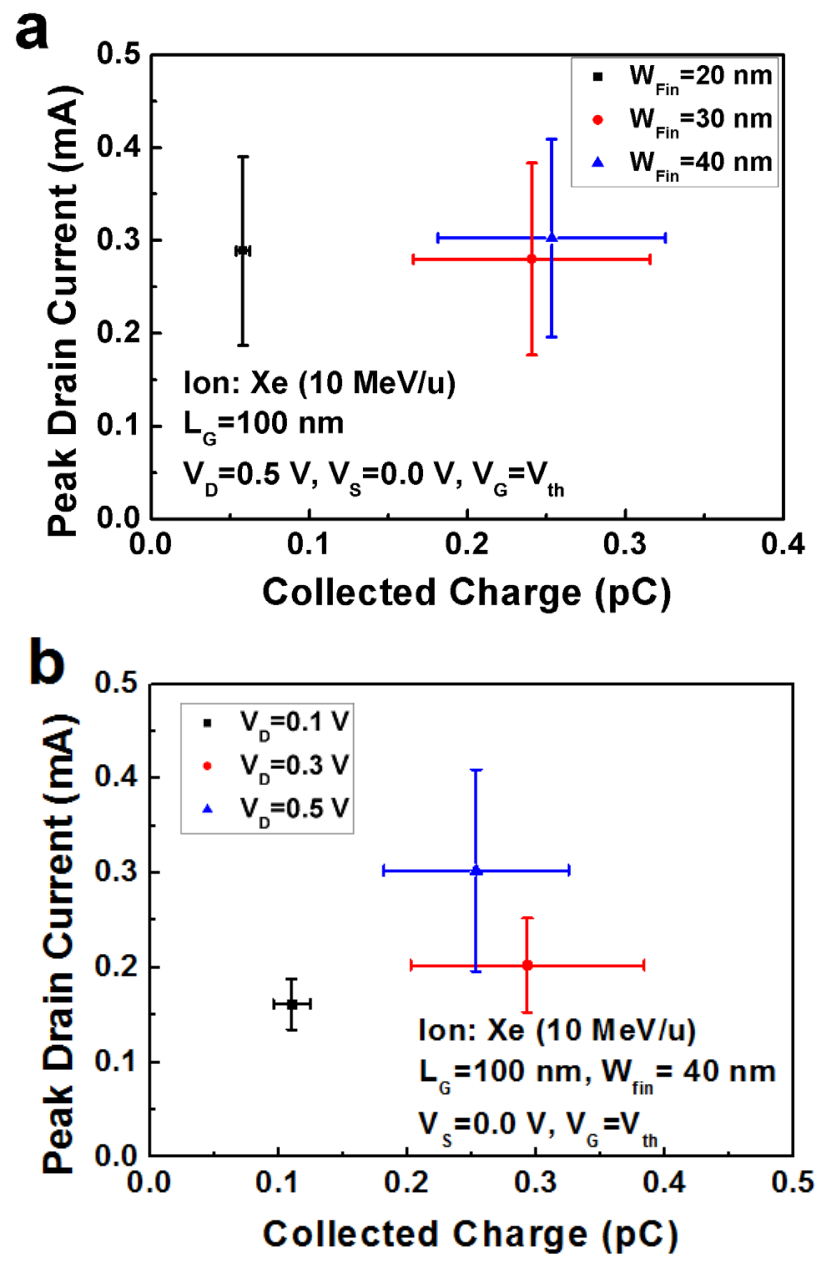

Fig. 8. Collected charge and peak drain current during Xe irradiation for devices with different fin width (a) and drain bias (b). Each data point is averaged from 50 transient events.

The SET cross-section has been calculated in the usual way:

$$
\sigma=\frac{N}{\phi \cdot t}
$$

where $\sigma$ is the SET cross-section, $N$ is the number of transients observed, $\phi$ is the ion flux, and $t$ is the observation time. Fig. 9 shows the SET cross-section for devices under $10 \mathrm{MeV} / \mathrm{u}$ Xenon irradiation. The minimum detectable event was $40 \mu \mathrm{A}$ peak current for all tests. Each cross-section result is averaged from two tests. The SET cross-section shows a strong dependence on fin width. Narrow fin devices show smaller cross-sections compared with wider fin devices. 

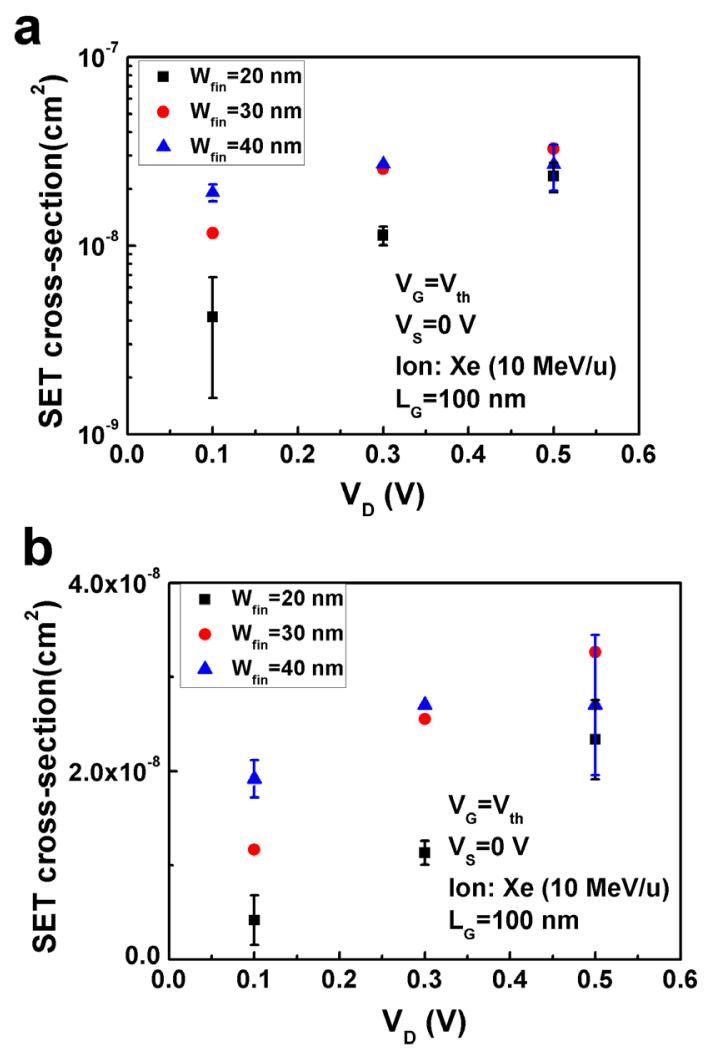

Fig. 9. SET cross-section vs. $V_{D S}$ for InGaAs FinFETs with different fin widths (a) log scale and (b) linear scale. Each data point is averaged from 50 transient events.
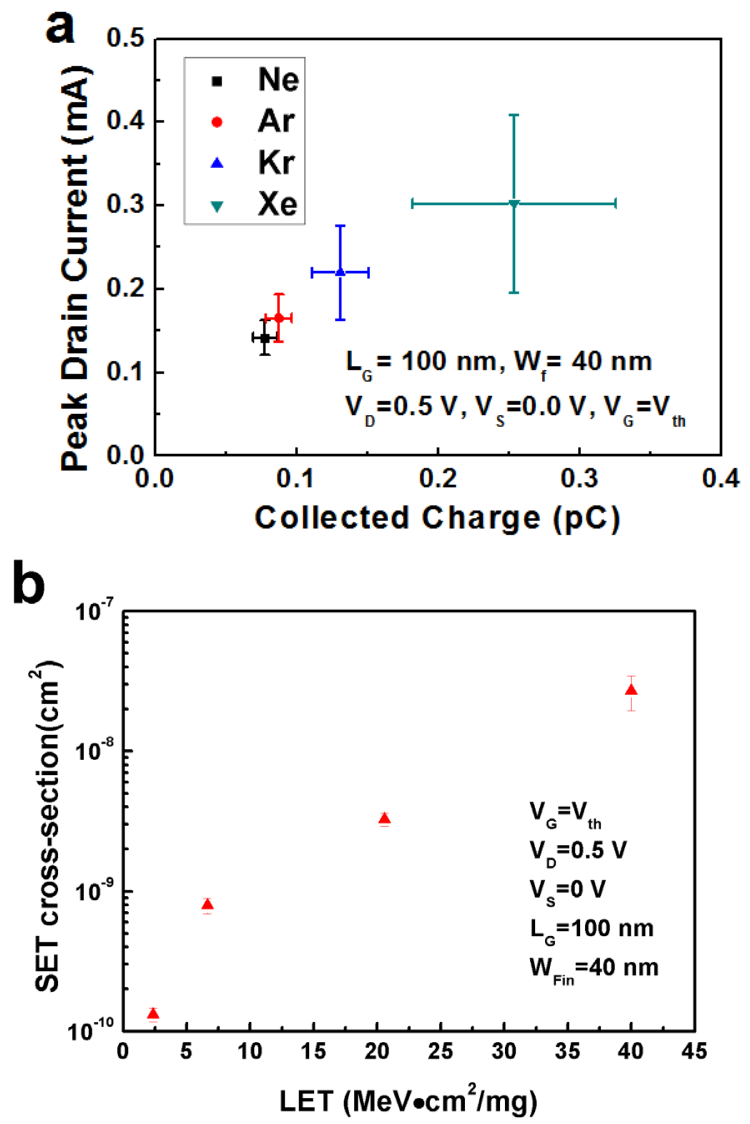

Fig. 10. (a) Collected charge, peak drain current and (b) SET cross-section for a $40 \mathrm{~nm}$ device with heavy-ion irradiation at different LET in $\operatorname{In}_{0.53} \mathrm{Ga}_{0.47} \mathrm{As}$.
By irradiating devices with various ions, LET effects on charge collection are investigated. Fig. 10a shows the peak drain current and collected charge for a $40 \mathrm{~nm}$ fin width device irradiated with ions of different LETs. As expected, more charge is collected for particles with higher LETs, and thus higher peak currents. Fig $10 \mathrm{~b}$ shows the SET cross-section for a $40 \mathrm{~nm}$ fin width device irradiated with different ions. The SET cross-section increases as LET increases, and no saturation cross-section is observed. This response is not surprising because the area of the devices $\left(1.8 \times 10^{-7} \mathrm{~cm}^{2}\right)$ is larger than the observed cross-sections.

\section{TCAD SIMULATIONS}

\section{A. Geometric effect}

3-D TCAD simulations were performed with Sentaurus to investigate charge collection mechanisms for both pulsed-laser and heavy ion irradiation [19]. The simulated devices have gate lengths of $100 \mathrm{~nm}$, with different fin widths. Only single fin structures were simulated, which is sufficient to understand the trends in charge collection. The deposited charge in the simulations was $75 \mathrm{fC} / \mu \mathrm{m}$ over a distance of $8 \mu \mathrm{m}$, which is qualitatively similar to that produced by pulsed-laser irradiation. The characteristic width of the Gaussian distribution is $50 \mathrm{~nm}$ (fin spacing is much larger than this value). Charge was injected from the top of the gate to the substrate, centered at the middle of the channel. The Gaussian temporal distribution was centered at $1 \mathrm{~ns}$ with a characteristic time of 2 ps. For simplicity, optical absorption (only present in the $40 \mathrm{~nm} \operatorname{In}_{0.53} \mathrm{Ga}_{0.47} \mathrm{As}$ layer) was neglected in these TCAD simulations.

Fig. 11a shows simulated drain transient currents for devices at a gate bias of $-0.6 \mathrm{~V}$. The wider fin devices show both higher peak current and larger charge collection. Fig. 11b shows how the potential along the channel, represented by the conduction band energy, evolves after the charge is deposited. At $1 \mathrm{~ns}$, as the charge is deposited, the barrier from the source to drain has dropped significantly due to hole accumulation at the source side, corresponding to the rising edge at $1 \mathrm{~ns}$ in Fig. 11a. After about $5 \mathrm{~ns}$, the barrier recovers to the initial value, which makes the transient current disappear, as shown in Fig. 11a. These results are consistent with the pulsed-laser results, where reduced peak current and charge are observed for the narrowest fin device. These results also are qualitatively consistent with trends observed in SEE tests on Intel's 14-nm second-generation tri-gate technology, in which the soft error rate (SER) improvements for narrower fin technology were also primarily attributed to reduced charge collection due to smaller collection volumes [20].

\section{B. Channel modulation by the substrate}

The generated charge may accumulate underneath the channel during the transient, acting as a back gate and modulating the channel. The electrostatics of the effect are similar to what has been examined for TID effects in FinFETs, which show that the modulation of channel potential by charge beneath the fin is stronger for wider fin devices. Narrow fin devices have better control from the two side gates [21]-[23]. 
Fig. 12a shows a TCAD simulation on $20 \mathrm{~nm}$ fin-width devices with different amounts of charge in the silicon delta doping (red) region underneath the channel. As the charge density beneath the fin increases from $2 \times 10^{19} / \mathrm{cm}^{3}$ to $2 \times 10^{21} / \mathrm{cm}^{3}$, the electron density in the channel is strongly affected. For wider fin devices, the charge acts as a back gate, modulating the charge density in the channel. Narrower fin devices are less affected by the back gate modulation effect due to its shorter back gate length which is equivalent to the fin width, and the channels are more strongly modulated by the two side gates.
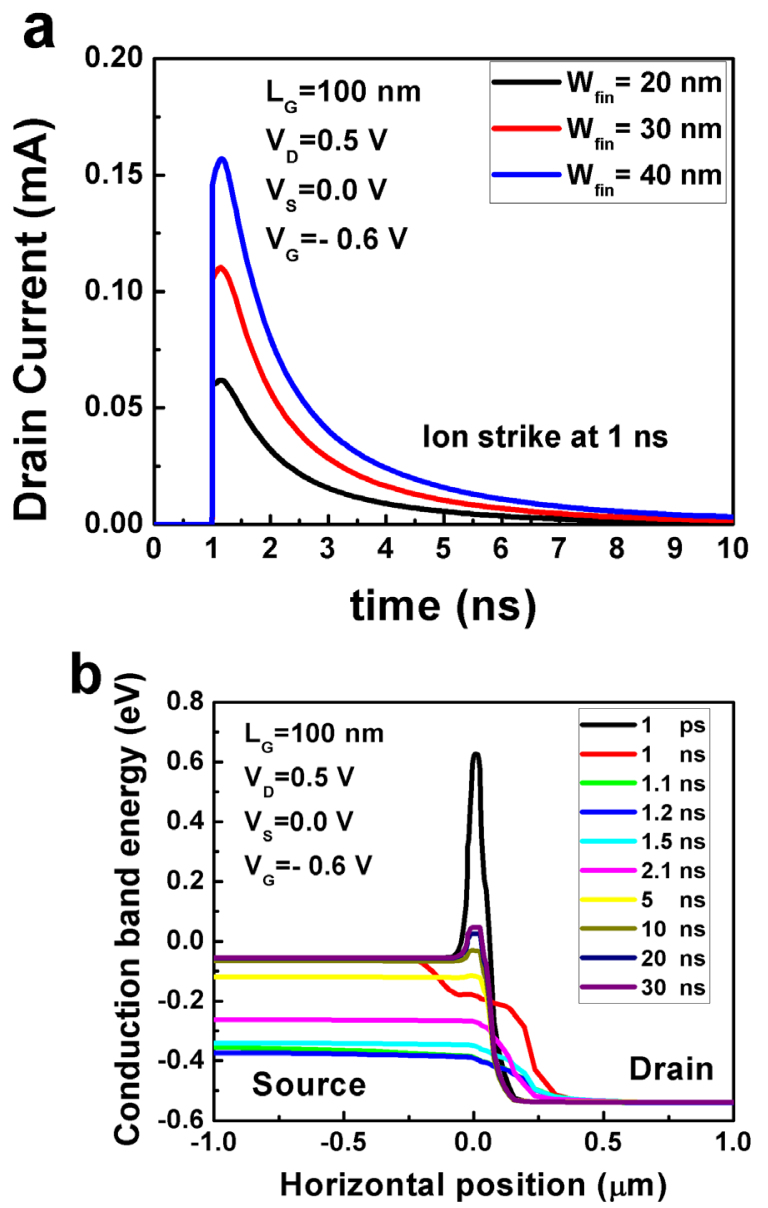

Fig. 11. (a) Drain current transients for InGaAs FinFETs of different fin widths from TCAD simulation, (b) conduction band energy evolution along the channel after charge deposition.

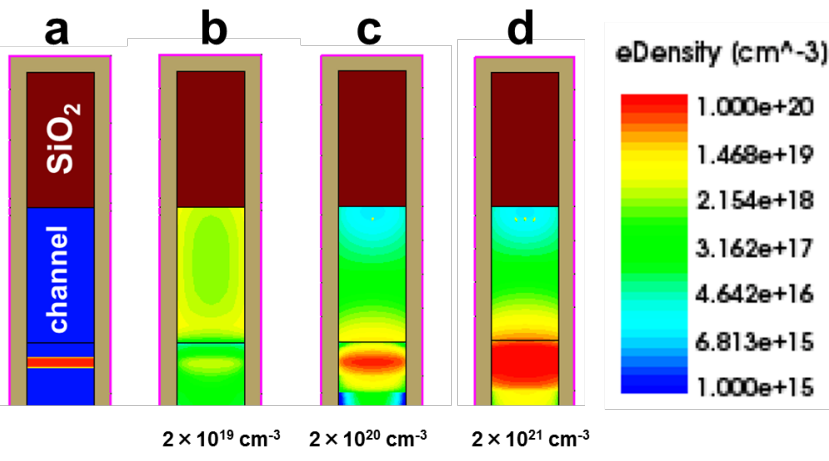

Fig. 12. Electron density distribution (b,c,d) for $20 \mathrm{~nm}$ fin-width devices with different doping in the red region (a). $\mathrm{V}_{\mathrm{G}}=0.6 \mathrm{~V}, \mathrm{~V}_{\mathrm{D}}=0.5 \mathrm{~V}, \mathrm{~V}_{\mathrm{D}}=0 \mathrm{~V}$.

\section{Optical Simulations}

Both pulsed-laser and heavy-ion experiments show that the charge collection is not linearly related to fin width. The difference in peak current dependence between the laser testing and ion testing results suggests that other mechanisms affect charge collection that have not been previously considered. In particular, during pulsed-laser tests, the optical intensity distribution is modified by optical interference effects, and the peak optical intensity may be enhanced inside the fin due to plasmonic effects resulting from the nanoscale metal-dielectric-metal geometry of the fins.

To evaluate the potential effects of optical phenomena that occur in laser testing but not ion testing, optical simulations were carried out using the Lumerical optical design tool and a simplified model system. Fig. 13 shows initial optical simulation results on a simplified FinFET model system with different fin widths, conducted using two-dimensional FDTD analysis [12]. These simulations provide intuition on the relative importance of optical phenomena and geometric size-based effects in nanoelectronic devices. In the simulations, for simplicity, the fins are modeled to be $\mathrm{Si}$ and the metal contact on the top is selected to be Al. Laser excitation is modeled as a Gaussian source, polarized orthogonal to the vertical dimension of the fin and propagating from the bottom of the structure toward the fin. The wavelength of the source in the simulations is $1250 \mathrm{~nm}$ with a $1 \mu \mathrm{m}$ radius focused at the bottom of the fins; the absorption coefficient of $\mathrm{Si}$ is $\sim 0.0181 \mathrm{~m}^{-1}$ [12], [24]. This model system evaluates the optical response of a fin to sub-band-gap irradiation [25], [26], as is the case for the majority of the fin $(180 \mathrm{~nm}$ out of $220 \mathrm{~nm})$ in the device under test. In this first-order approximation, the integrated $E^{2}$ intensity in the fins is assumed to be proportional to the energy absorbed in the fins, which is in turn related to the charge generation in the fins.

Optical interference and plasmonic enhancement are revealed in Fig. 13 by the periodic oscillations of the $E^{2}$ intensity and by the peak $E^{2}$ intensity values, respectively. The change in the spacing of the $E^{2}$ intensity oscillations for the different fin widths can be explained by traditional optical interference effects between the incident and reflected light waves [27]. In a more realistic three-dimensional model that incorporates sidewall surface roughness, multiple reflections may build up inside the fin, modifying the interference pattern and potentially enhancing the electric field inside the fin. The higher peak $E^{2}$ intensity in the narrower fins revealed by examination of the color bar scale in Fig. 13 is consistent with plasmonic enhancement in nanoscale metal-dielectric-metal structures in which the enhancement scales inversely with the thickness of the dielectric layer [28], [29]. The strongest plasmonic enhancements in such structures typically occur for gaps of less than $10 \mathrm{~nm}$ between metal features; hence plasmonic effects are likely to be weak in the tested FinFETs. Therefore, while plasmonic enhancement likely increases the $E^{2}$ intensity and hence the expected charge generation by SPA per unit area in the $20 \mathrm{~nm}$ fin, the geometric effect is dominant and the increased area in the larger fins more than compensates for the weak plasmonic effect. Hence, the integrated $E^{2}$ intensity in the 
$40 \mathrm{~nm}$ fin is larger than that in the $30 \mathrm{~nm}$ fin, which in turn is larger than that in the $20 \mathrm{~nm}$ fin in both the optical simulations and laser testing experiments. Moreover, multiple reflections are likely to lead to a significant increase in local e-h pair density in the smallest devices, leading to an enhanced recombination rate. This may also contribute to the reduced charge collection in the $20 \mathrm{~nm}$ fin devices. Based on these general considerations, we believe that optical phenomena will play a significant role in laser-based testing of FinFET technology at the $14 \mathrm{~nm}$ node and below. We conclude that these initial simulations in a simple model system are qualitatively consistent with charge collection trends in this work; a more detailed analysis will be the topic of future work.
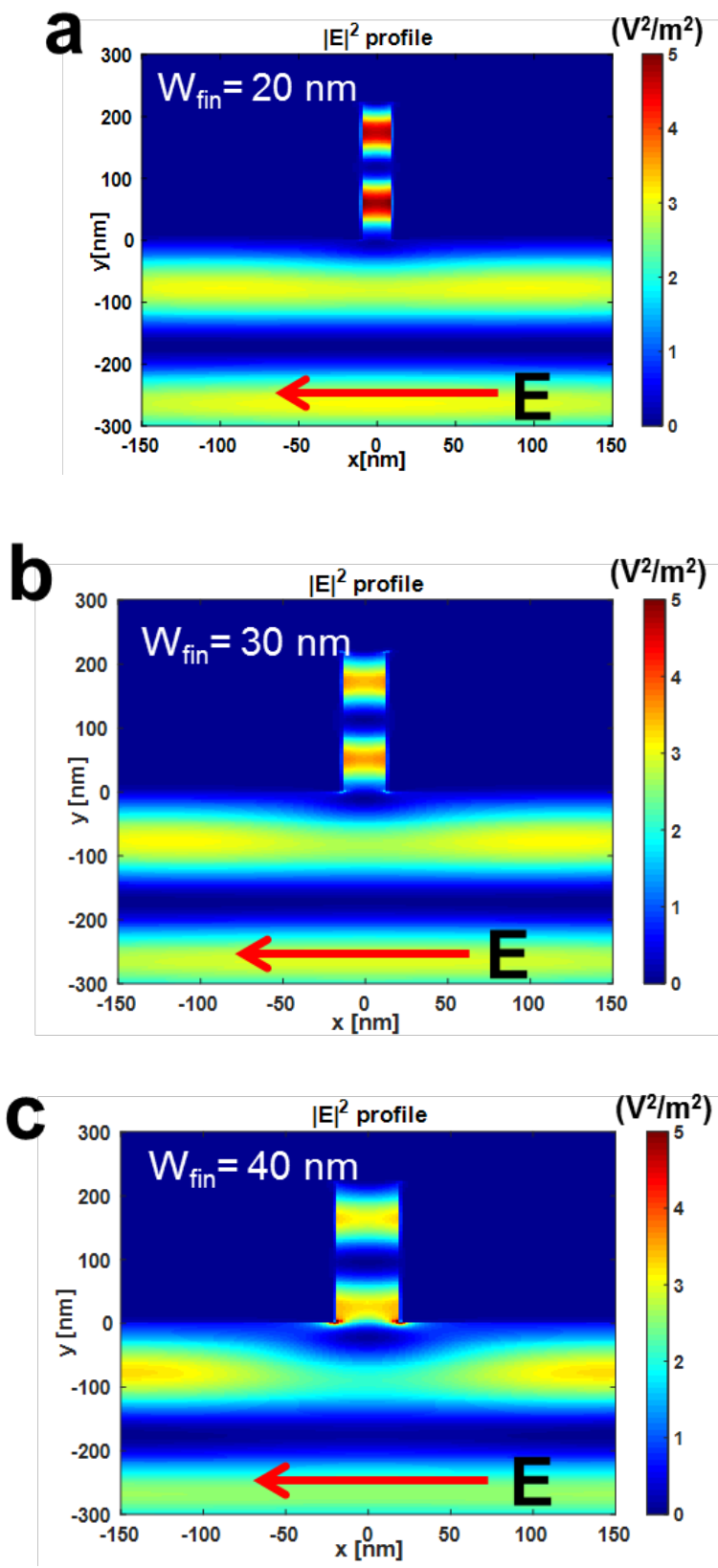

Fig. 13. Optical $\left(E^{2}\right)$ field distribution calculated by $2 \mathrm{D}$ Lumerical simulations for simplified silicon FinFETs with different fin width.

\section{SUMMARY}

This paper describes the SET response of InGaAs FinFETs with different fin widths. For both pulsed-laser irradiation and heavy-ion irradiation, wider fin devices collect more charge. The SETs exhibit similar drain bias dependence for both types of irradiation. Larger drain bias increases the electric field on the drain side, which produces higher peak drain current and more collected charge. The SET cross section also scales with fin width, and stronger scaling effects are observed for lower drain biases. The transient tails overlap for all devices, which is due to similar charge collection in the substrate for all device widths. The SET dependence on ion LET is also reported. Ions with higher LET generate more electron-hole pairs in the channel, and thus higher peak drain current, more collected charge, and larger SET cross-section.

3D TCAD simulations show that the enhanced charge collection of the wider fin devices is mainly due to larger geometric volumes. Charge accumulated underneath the fin functions as a back gate, which produces stronger channel modulation effects for wider fin devices. Lumerical simulations on a simple model system are consistent with observed trends of laser-induced charge collection with fin width, and suggest that optical phenomena including plasmonic effects and enhanced e-h pair recombination due to multiple reflections within confined, multi-gate structures will play an increasingly important role for pulsed-laser induced SET tests of advanced nanoelectronic technologies. These include emerging FinFETs and gate-all-around (GAA) devices, and thus is an important consideration in testing of nanoscale devices.

\section{ACKNOWLEDGMENT}

The authors thank Dennis Ball, Pan Wang and Peng Wang for helpful discussions.

\section{REFERENCES}

[1] J. A. del Alamo, "Nanometre-scale electronics with III-V compound semiconductors," Nature, vol. 479, no. 7373, pp. 317-323, Nov. 2011.

[2] T. R. Weatherford and W. T. Anderson, Jr, "Historical perspective on radiation effects in III-V devices," IEEE Trans. Nucl. Sci., vol. 50, no. 3, pp. 704-710, Jun. 2003.

[3] J. M. Borrego, R. J. Gutmann, S. B. Moghe, and M. J. Chudzicki, "Radiation effects on GaAs MESFETs," IEEE Trans. Nucl. Sci., vol. 25, no. 6, pp. 1436-1443, Dec. 1978.

[4] I. K. Samsel, E. X. Zhang, N. C. Hooten, et al., "Charge collection mechanisms in $\mathrm{AlGaN} / \mathrm{GaNMOS}$ high electron mobility transistors," IEEE Trans. Nucl. Sci, vol. 60, no. 6, pp. 4439-4445, Dec. 2013.

[5] D. McMorrow, J. B. Boos, A. R. Knudson, et al., "Transient response of III-V field-effect transistors to heavy-ion irradiation," IEEE Trans. Nucl. Sci, vol. 51, no. 6, pp. 3324-3331, Dec. 2004.

[6] D. McMorrow, T. R. Weatherford, S. Buchner, et al., "Single event phenomena in GaAs devices and circuits," IEEE Trans. Nucl. Sci., vol. 43, no. 2, pp. 628-644, Apr. 1996.

[7] S. DasGupta, D. McMorrow, R. A. Reed, R. D. Schrimpf, and J. B. Boos, "Gate bias dependence of single event charge collection in AlSb/InAs HEMTs," IEEE Trans. Nucl. Sci., vol. 57, no. 4, pp. 1856-1860, Aug. 2010.

[8] K. Ni, E. X. Zhang, N. C. Hooten, et al., "Single event transient response of InGaAs MOSFETs," IEEE, Trans. Nucl. Sci., vol. 61, pp. 3550-3556, Dec. 2014.

[9] K. Ni, J. A. Smith, M. Barth, et al., "Soft error evaluation for InGaAs and Ge complementary FinFETs," p. P-23, IEEE Device Research Conf., Notre Dame, IN, June 25-28, 2017.

[10] https://newsroom.intel.com. 
[11] K. Ni, A. L. Sternberg, E. X. Zhang, et al., "Understanding charge collection mechanisms in InGaAs FinFETs using high-speed pulsed laser testing with tunable wavelength," IEEE Trans. Nucl. Sci., vol. 64, no. 8, pp. 2069-2078, Aug. 2017.

[12] Lumerical: https://www.lumerical.com/tcad-products/fdtd/ .

[13] A. Vardi, and J. A. del Alamo, "Sub-10-nm fin-width self-aligned InGaAs FinFETs" IEEE Electron Device Lett., vol. 37, no. 9, pp. 1104-1107, Sep. 2016.

[14] A. Vardi, X. Zhao, and J. A. del Alamo, "Quantum-size effects in sub 10-nm fin width InGaAs FinFET," IEEE Int. Electron Device Meeting (IEDM), pp. 31.3.1-31.3.4, 2015.

[15] N. C. Hooten, W. G. Bennett, L. D. Edmonds, et al., "The impact of depletion region potential modulation on ion-induced current transient response," IEEE Trans. Nucl. Sci., vol. 60, no. 6, pp. 4150-4158, Dec. 2013.

[16] F. El-Mamouni, E. X. Zhang, N. D. Pate, et al., "Laser and heavy-ion induced charge collection in bulk FinFETs," IEEE Trans. Nucl. Sci., vol. 58, no. 6, pp. 2563-2569, Dec. 2011.

[17] F. El-Mamouni, E. X. Zhang, D. R. Ball, et al., "Heavy-ion-induced current transients in bulk and SOI FinFETs," IEEE Trans. Nucl. Sci., vol. 59, pp. 2674-2681, Dec. 2012.

[18] E. X. Zhang, I. K. Samsel, N. C. Hooten, et al., "Heavy ion and laser induced charge collection in SiGe channel $p$ MOSFETs," IEEE Trans. Nucl. Sci., vol. 61, no. 6, pp. 3187-3192, Dec. 2014.

[19] TCAD: https://www.synopsys.com.

[20] N. Seifert, S. Jahinuzzaman, J. Velamala, et al., "Soft error rate improvements in 14-nm technology featuring second-generation 3D tri-gate transistors," IEEE Trans. Nucl. Sci., vol. 62, no. 6, pp. 2570-2577, Dec. 2015.

[21] F. El-Mamouni, E. X. Zhang, R. D. Schrimpf, D. M. Fleetwood, R. A. Reed, S. Cristoloveanu, and W. Xiong, "Fin-width dependence of ionizing radiation-induced subthreshold-swing degradation in $100-\mathrm{nm}$ gate-length finFETs," IEEE Trans. Nucl. Sci., vol. 56, no. 6, pp. 32503255, Dec. 2009.

[22] J. Song, B. K. Choi, E. X. Zhang, et al., "Fin width and bias dependence of the response of triple-gate MOSFETs to total dose irradiation," IEEE Trans. Nucl. Sci., vol. 58, no. 6, pp. 2871-2875, Nov. 2011.

[23] E. Simoen, M. Gaillardin, P. Paillet, et al., "Radiation effects in advanced multiple gate and silicon-on-insulator transistors," IEEE Trans. Nucl. Sci., vol. 60, no. 3, pp. 1970-1991, May 2013.

[24] E. D. Palik, Ed., Handbook of Optical Constants of Solids I, Academic Press, San Diego, CA, 1997, pp. 547-569.

[25] J. S. Melinger, S. Buchner, D. McMorrow, W. J. Stapor, T. R. Weatherford, and A. B. Campbell, "Critical evaluation of the pulsed laser method for single event effects testing and fundamental studies," IEEE Trans. Nucl. Sci., vol. 41, no. 6, pp. 2574-2584, Dec. 1994.

[26] D. McMorrow, W. T. Lotshaw, J. S. Melinger, S. Buchner, and R. L. Pease, "Subbandgap laser-induced single event effects: carrier generation via two photon absorption," IEEE Trans. Nucl. Sci., vol. 49, no. 6, pp. 3002-3008, Dec. 2002.

[27] E. Hecht, Optics. San Francisco, CA: Addison Wesley, 2002.

[28] C. Ciracì, R. T. Hill, J. J. Mock, et al., "Probing the ultimate limits of plasmonic enhancement," Science, vol. 337, pp. 1072-1074, Aug. 2012.

[29] M. Miyata, A. Holsteen, Y. Nagasaki, et al., "Gap plasmon resonance in a suspended plasmonic nanowire coupled to a metallic substrate," Nano Lett., vol. 15, pp. 5609-5616, July 2015. 